\title{
ON THE MINIMAL SPACE OF SURJECTIVITY QUESTION FOR LINEAR TRANSFORMATIONS ON VECTOR SPACES WITH APPLICATIONS TO SURJECTIVITY OF DIFFERENTIAL OPERATORS ON LOCALLY CONVEX SPACES
}

\author{
D.K. COHOON
}

\author{
Mathematics Department 038-16 \\ Temple University \\ Philadelphia, Pennsylvania 19122 \\ (Received March 6, 1986)
}

ABSTRACT. We use transfinite induction to show that if $L$ is an epimorphism of a vector space $V$ and maps a vector subspace $W$ of $V$ into a proper subspace of itself, then there is a smallest subspace $E$ of $V$ containing $W$ such that $L(E)=E$ (or a minimal space of surjectivity or solvability) and we give examples where there are infinitely many distinct minimal spaces of solvability. We produce an example showing that if $L_{1}$ and $L_{2}$ are two epimorphisms of a vector space $V$ which are endomorphisms of a proper subspace $W$ of $V$ such that $L_{1}(W) \cap L_{2}(W)$ is a proper subspace of $W$, then the re may not exist a smallest subspace $E$ of $V$ containing $W$ such that $L_{1}(E)=E=L_{2}(E)$. While no nonconstant linear partial differential operator maps the field of meromorphic functions onto itself, we construct a locally convex topological vector space of formal power series containing the meromorphic functions such that every linear partial differential operator with constant coefficients maps this space linearly and continuously onto itself. Furthermore, we show that algebraically there is for every linear partial differential operator $P(D)$ with constant coefficients a smallest extension $E$ of the meromorphic functions in $n$ complex variables, where

$D=\left(\frac{\partial}{\partial z_{1}}, \ldots, \frac{\partial}{\partial z_{n}}\right)$, with the property that for every $f$ in $E$, there is a $u$ in $E$ such that $P(D) u=f$.

KEY WORDS AND PHRASES. Axiom of choice, category theory, epimorphisms of vector spaces, extensions of vector space endomorphisms, linear transformations, partial differential operators with constant coefficients, transpose of a linear partial differential operator, meromorphic functions, formal power series, global solvability of partial differential equations.

1980 AMS SUBJECT CLASSIFICATION CODE: 4690043004201660162635801505. 


\section{INTRODUCTION.}

A straightforward argument using transfinite induction and the axiom of choice will show that if $L$ is a linear transformation of the vector space $V$ over the field $F$ onto itself, and $W$ is a subspace of $V$ such that $L(W)$ is a proper subset of $W$, then there is a subspace $E$ of $V$ containing $W$ such that $L(E)=E$ and such that if $F$ is any proper subspace of $E$ containing $W$, then $L(F) \neq F$. That is to say there is a smallest extension $E$ of $W$ in which the inhomogeneous equation

$$
L u=f
$$

has a solution $u$ in $E$ for every $f$ in $E$.

In section two of this paper we show that there is a vector space $V$, a subspace $W$ of $V$, a pair of linear transformations $L_{1}$ and $L_{2}$ of $V$ onto itself such that $L_{1}(W) \cap L_{2}(W)$ is a proper subspace of $W$ and such that if $E$ is any vector space containing $W$ and contained in $V$ such that $L_{1}(E)=L_{2}(E)=E$, then there is also a proper subspace $F$ of $E$ containing $W$ such that $L_{1}(F)=L_{2}(F)=F$. Thus the minimal space of surjectivity question for families of mappings is not solvable.

If $C$ is a category whose objects are sets, possibly equipped with some structure, and whose morphisms are mappings between the sets, which preserve the structure, then we can define the minimal space of surjectivity question as follows. Let $C$ be such a category. Let $V$ be an object in $C$ and let $W$ be a subobject of $V$, a subset of $V$ which has the structure (if any) induced by that of $V$. Let $L$ be a mapping that is an epimorphism of $V$ in the sense that (e.g. Northcott [1], chapter III) $V$ is the unique object in the category of vector spaces and linear transformations such that $I V L$ and $L I_{V}$ are defined where $I_{V}$ is the identity mapping of $V$ and $L(V)=V$. Further assume that if $U$ is any subobject of $V$, then the restriction of $L$ to $U$ defines a morphism of the category whose range can be any subobject of $V$ containing $U$. Then we say $E$ is a solution of the minimal space of surjectivity problem defined by the triple $(V, W, L)$ satisfying the preceding conditions if $L(E)=E, L(W)$ is a proper subspace of $W$, and if $F$ is any subspace of $E$ containing $W$, then $L(F) \neq F$. If there is a triple for which there is no solution to the minimal space of surjectivity problem we say that for the category the MSS question has a negative answer. If there is a triple $(V, W, L)$ satisfying the above conditions for which the MSS problem does not have a unique answer, we say that there is nonuniqueness for the MSS question for the category.

In section 3 of this paper we show that the MSS question has a positive answer for the category of vector spaces and linear transformations, but in section 4 we show that we have nonuniqueness in this category.

In section 5 of this paper we show, for every nonzero linear partial differential operator with constant coefficients, the existence of a smallest extension of the meromorphic functions on which the operator is an epimorphism. We do this by exhibiting a locally convex topolgoical vector space containing the meromorphic functions on which every linear partial differential operator is an epimorphism and applying the results of the previous sections. 


\section{NONEXISTENCE OF A MSS FOR SOME FAMILIES OF EPIMORPHISMS.}

It can be shown that if $V$ is a vector space, $W$ is a subspace of $V$, and $L$ is an epimorphism of $V$ such that $L(W)$ is a proper subset of $W$, then there is a subspace $E$ of $V$ containing $W$ which is minimal with respect to surjectivity in the sense that $L(E)=E$, but if $U$ is any proper subspace of $E$ containing $W$, then $L(U)$ does not contain $U$. It seems natural to ask the same question for families of linear transformations.

MSS Question for Families of Mappings. Let $V$ be a vector space. Let $W$ be a subspace of $V$. Let $F$ be a family of epimorphisms of $V$ such that $L(W) \subset W$ for all $L$ in $F$ and $L(W) \neq W$ for at least one $L$ in $F$. Does there exist a subspace $E$ of $V$ containing $W$ such that $L(E)=E$ for every $L$ in $F$ having the additional property that if $U$ is any subspace of $E$ containing $W$, then $L(U) \neq U$ for some $L$ in $E$ if $U \neq E$.

The following theorem shows that the MSS question for families of linear transformations fails in general to have a positive answer even if $F$ contains only two mappings.

Theorem 2.1. There exists a vector space $V$, two epimorphisms $L_{1}$ and $L_{2}$ of $V$, a subspace $W$ of $V$ such that $L_{k}(W) \subset W$ for $k=1,2$, such that $L_{1}(W) \cap L_{2}(W)$ is a proper subset of $W$, and having the additional property that if $E$ is any subspace of $V$ containing $W$ such that $L_{k}(E)=E$ for $k=1,2$ then there is a proper subspace $U$ of $E$ containing $W$ such that $L_{k}(U)=U$ for $k=1,2$.

Proof of Theorem 2.1. Let $N$ denote the set of nonnegative integers. Let $Q$ denote the set of all nonzero integer powers of the prime $q$. Let $F$ denote an arbitrary field. For convenience we introduce the following.

Definition 2.1. If $S$ is a set without a topology and $\Psi: S+F$ is a mapping from the set $S$ into a field $F$, then the support of $\psi$ is defined by the rule,

$$
\operatorname{supp}(\Psi)=\{j \in S: \Psi(j) \neq 0\}
$$

We let $v_{0}$ denote the vector space of mappings from $\{0\}$ into the field $F$. For every positive integer $k$ we let $V_{k}$ denote the vector space of mappings from $Q$ into $F$ whose support is a finite subset of $Q$. Let $P: V_{k} \rightarrow V_{k}$ denote the projector onto the space of functions whose supports are subsets of

$$
N_{q}=\left\{q^{-n}: n=1,2,3, \ldots\right\} \text {. }
$$

Let $T: P\left(V_{k}\right) \rightarrow P\left(V_{k}\right)$ be a linear transformation defined by the rule

$$
T\left(P \psi_{k}\right)\left(q^{-2 n}\right)=P \psi_{k}\left(q^{-n}\right)
$$

and

$$
T\left(P \Psi_{k}\right)\left(q^{-(2 n-1)}\right)=0
$$

for all positive integers $n$ and all functions $\Psi_{k}$ in $V_{k}$. 
Lemma 2.1. If ${ }^{\psi}$ is a function in $P\left(V_{k}\right)$ whose support is precisely $\left\{q^{-n_{1}}, q^{-I_{2}}\right.$, $\ldots, a^{-n} r$, then the support of $T \Psi$ is exactly $\left\{q^{-2 n_{1}}, q^{-2 n_{2}}, \ldots, q^{-2 n_{r}} r_{\}}\right.$.

Proof of Lemma 2.1. Suppose $q^{-2 n}$ were in the support of $T \psi$. Then by definition $n$ must be one of $\left\{n_{1}, n_{2}, \ldots, n_{r}\right\}$.

As a corollary of Lemma 2.1 we observe that $\operatorname{Ker}(T)$ is trivial.

Let $\tilde{P}: V_{k} \rightarrow V_{k}$ denote the projector defined by the rule

$$
\tilde{p} \Psi\left(q^{2 n}\right)=\Psi\left(q^{2 n}\right)
$$

for all positive integers $n$, and

$$
\tilde{P} \Psi\left(q^{m}\right)=0
$$

if $m$ is an integer that is not equal to $2 n$ for some positive integer $n$.

Let. $B: v_{1} \rightarrow v_{0}$ be defined by the rule,

$$
B \Psi=\Phi,
$$

where

$$
\Phi(0)=\sum_{k=0}^{\infty} \Psi\left(q^{2 n+1}\right) .
$$

Define a mapping,

$$
S: V_{k} \rightarrow(I-P) V_{k}
$$

by the rule,

$$
\begin{aligned}
S \Psi\left(q^{2 n}\right) & =\Psi\left(q^{n}\right), \\
S \Psi\left(q^{2 n-1}\right) & =\Psi\left(q^{-n}\right),
\end{aligned}
$$

and

$$
S \Psi\left(q^{-n}\right)=0
$$

for all positive integers $n$.

Let $V$ be the vector space over $F$ defined to be the set of all

$$
\left(\psi_{0}, \psi_{1}, \ldots, \psi_{k}, \ldots\right)
$$

where $\psi_{k}$ is a member of $V_{k}$ for all nonnegative integers $k$ and $\psi_{k}$ is identically zero for all but a finite number of nonnegative integers $k$, and let $w$ denote the space of

$$
w=\left(\psi_{0}, \Phi_{1}, \ldots, 0, \ldots\right)
$$


where $\psi_{0}$ is a member of $V_{0}$ and $\Phi_{1}$ is a member of $\bar{P}\left(V_{1}\right) \cap \operatorname{Ker}(B)$ where

$$
\bar{P}=I-P-\tilde{P} \text {. }
$$

Define a mapping $L_{1}: V \rightarrow V$ by the rule,

$$
L_{1}\left(\psi_{0}, \psi_{1}, \ldots, \psi_{n}, \ldots\right)=\left(B\left(\bar{P}_{1}\right), T P \psi_{1}+P_{\psi}{ }_{1}+\psi_{2}, \ldots, T P \psi_{n}+P_{\psi} \psi_{n}+\psi_{n+1}, \ldots\right)
$$

Define a mapping $L_{2}: V \rightarrow V$ by the rule

$$
L_{2}\left(\psi_{0}, \psi_{1}, \psi_{2}, \ldots, \psi_{n}, \ldots\right)=\left(\psi_{0}, S \psi_{1}+\psi_{2}, \ldots, S \Psi_{n}+\Psi_{n+1}, \ldots\right)
$$

Lemma 2.2. $L_{k}: V \rightarrow V$ is an epimorphism for $k=1,2$, and $L_{2}: V \rightarrow V$ is an isomorphism.

Proof. That each $L_{k}$ is an epimorphism is obvious since $V_{0}$ is one dimensional and $\Psi_{n+1}$ covers the part of the $n$th coordinate space not covered by $T P \Psi_{n}+P_{\Psi_{n}}$ or $S \Psi_{n}$, respectively. Also if each $S \psi_{n}+\psi_{n+1}$ is identically zero this implies that each $\psi_{n}$ is identically zero, if $\psi_{n+1}=0$, since $S: V_{n} \rightarrow(I-P) V_{n}$ is an isomorphism.

Now let us construct a space of surjectivity, $E$, for the operators $L_{1}$ and $L_{2}$ which contains $W$. Let $\left(\Psi_{0}, 0,0, \ldots\right)$ denote a nonzero member of $W$. Then there exists a vector

$$
\left(\tilde{\Psi}_{0}, \psi_{1}, \ldots, \Psi_{n}, 0,0, \ldots\right)=\tilde{v} \text { in } v
$$

such that

$$
L_{1} \tilde{v}=\left(\Psi_{0}, 0, \ldots, 0, \ldots\right)
$$

Since $\left(-\tilde{\psi}_{0}, 0, \ldots, 0, \ldots\right)$ is in $W$ and $E$ is a vector space we conclude that

$$
v=\left(0, \Psi_{1}, \Psi_{2}, \ldots, \Psi_{n}, 0,0, \ldots\right),
$$

it in $E$ and $L_{1} v=L_{1} \tilde{v}$. Now suppose that $n>1$. Then the $n$th entry of $L_{1}\left(0, \psi_{1}, \ldots, \psi_{n}, 0,0, \ldots\right)$ is $T P \psi_{n}+\tilde{P}_{n}$. Since $T: P V_{n} \rightarrow P V_{n}$ is a monomorphism by Lemma 2.1 we conclude that $P_{n}{ }_{n}$ and $P_{\Psi_{n}}$ are identically zero. Now we know that $P \Psi_{n-1}+$ $P_{\Psi_{n-1}}+\Psi_{n}$ is identically zero. Thus, the fact that $(P+P)\left(P \Psi_{n-1}+P_{n}{ }_{n}+\Psi_{n}\right)$ is identically zero implies that $P \Psi_{n-1}+P_{\Psi_{n-1}}$ is identically zero. Hence, $\Psi_{n}$ is identically zero. Since $n$ represents an arbitrary integer larger than 2 , we conclude that the vector $v$ defined in (2.15) is of the form

$$
v=\left(0, \Psi_{1}, 0,0, \ldots\right)
$$

where $P_{1}$ and $P_{\Psi}$ are identically zero. We conclude that

$$
\operatorname{supp}\left(\Psi_{1}\right)=\left\{q^{2 n_{k}-1}: k=1,2, \ldots, r\right\}
$$

where $0<n_{1}<n_{2}<\ldots<n_{k}<n_{k+1}<\ldots<n_{r}$. Presumably $\psi_{0}$ is not identically zero and, consequently, $\Psi_{1} \notin \operatorname{Ker}(B)$. Let $\operatorname{supp}\left(\psi_{1}\right)$ denote the support of $\Psi_{1}$. Then we 
observe that $L_{2} v \in E$ and that there is some $v_{1}$ in $E$ such that $L_{2} v_{1}=v=\left(0, \psi_{1}, 0, \ldots\right)$. We observe that $L_{2}$ is a one-to-one mappirig. Hence $L_{2}^{-1}(\{v\})$ contains only a single element. Observe that if the support of $\psi_{1}$ is defined by (2.16), then

$$
L_{2}^{-1}(v)=\left(0, \Phi_{1}, 0,0, \ldots, 0, \ldots\right)
$$

and

$$
\operatorname{supp}\left(\Phi_{1}\right)=\left\{q^{-n_{k}}: k=1,2, \ldots, r\right\}
$$

Now we observe that since $P_{\Phi_{1}}=\Phi_{1}$, that

$$
L_{1}^{n} v_{1}=\left(0, T^{n_{\Phi}}, 0, \ldots, 0, \ldots\right) \text {. }
$$

Notice that the support of $T^{n_{\Phi}}$ is given by

$$
\operatorname{supp}\left(T^{n_{\Phi}}\right)=\left\{q^{-2^{n} n_{1}}, q^{-2^{n} n_{2}}, \ldots, q^{-2^{n} n_{r}}\right\} .
$$

Now let us look at

$$
L_{2}\left(0, T^{n_{\Phi}}, 0, \ldots, 0, \ldots\right)=\left(0, S T^{n_{\Phi}}, 0, \ldots, 0, \ldots\right)
$$

Observe that the support of $S T^{n_{\Phi_{1}}}$ is given by

$$
\operatorname{supp}\left(S T^{n_{\Phi}}\right)=\left\{q^{22^{n} n_{1}-1}, \ldots, q^{22^{n} n_{r}-1}\right\} .
$$

Observe that

$$
B\left(S T^{n_{\Phi}}\right)=\Psi_{0}
$$

Thus, we can say that the vector space generated by the elements we know to be in $E$ has the property that

$$
W \subset L_{1}(E) \cap L_{2}(E)
$$

Indeed

$$
L_{1}\left(0, S T^{n_{\Phi}}, 0, \ldots, 0, \ldots\right)=\left(\Psi_{0}, 0,0, \ldots, 0, \ldots\right)
$$

Now the support of $S^{m} S T^{n} \Phi_{1}$ is given by

$$
\operatorname{supp}\left(S^{m} S T^{n_{1}}\right)=\left\{q^{2^{m}\left(2 \cdot 2^{n} n_{1}-1\right)}, \ldots, q^{2^{m}\left(2 \cdot 2^{n} n_{r}-1\right)}\right\}
$$

Now there must be a vector $w$ in $E$ of the form

$$
w=\left(\phi_{0}, \phi_{1}, \phi_{2}, \ldots, \phi_{n}, \ldots\right)
$$


such that

$$
L_{1}(w)=\left(0, \Psi_{1}, 0, \ldots, 0, \ldots\right)
$$

Since $W$ is a vector space and $\left(-\phi_{0}, 0,0, \ldots, 0, \ldots\right)$ is in $W$ we may assume that $W$ is actually of the form $\left(0, \phi_{1}, \phi_{2}, \ldots, \phi_{n}, \ldots\right)$, where $\phi_{1} \varepsilon \operatorname{Ker}(B)$. But $\phi_{1} \varepsilon \operatorname{Ker}(B)$ and $(P+\tilde{P})_{1}=0$ implies that

$$
(0,-\phi, 0, \ldots, 0, \ldots) \in W
$$

Thus, we may assume that

$$
w=\left(0,0, \phi_{2}, \ldots, \phi_{n}, \ldots\right)
$$

We can show that if $n>2$, that $\phi_{n}$ is identically zero. Thus, we may assume that

$$
w=\left(0,0, \phi_{2}, 0, \ldots, 0, \ldots\right)
$$

Since $(P+\tilde{P}) \phi_{2}=0$ we deduce that $\phi_{2}=\psi_{1}$. Hence, we deduce that

$$
\left\{\left(0, \psi_{1}, 0, \ldots, 0, \ldots\right), \quad\left(0,0, \psi_{1}, 0, \ldots, 0, \ldots\right), \ldots\right\}
$$

are all contained in $E$. Thus, the set of functions in $E$ whose coordinate functions are in $\bar{P}(\operatorname{Ker}(B))$ or else are modulo a function in $\bar{P}(\operatorname{Ker}(B))$ a function with support sufficiently far out forms a proper subspace of $E$ which is mapped onto itself by $L_{1}$ and $\mathrm{L}_{2}$.

3. THE MINIMAL SPACE OF SURJECTIVITY PROBLEM FOR VECTOR

SPACES AND LINEAR TRANSFORMATIONS

Let $L$ be a linear transformation of a vector space $V$ onto itself, and let $W$ be a subspace of $V$ such that $L(W)$ is a proper subspace of $W$. The solvability of the MSS problem for the category of vector spaces and linear transformations is expressed in the following theorem.

Theorem 3.1. Let $L, V$, and $W$ be as defined in the introduction to this section. Then there is a subspace $E$ of $V$ containing $W$ such that $L(E)=E$ and such that if $E^{1}$ is a proper subspace of $E$ containing $W$, then $L\left(E^{1}\right) \neq E^{1}$.

Proof of Theorem 3.1. Let $W_{1}$ be a subspace of $W$ such that $W=L(W) \oplus W_{1}$. Let $B\left(W_{1}\right)$ be a bases for $W_{1}$. Let $E_{1}(W)$ be a set consisting of precisely one member from each of the sets in the family

$$
\left\{L^{-1}(w): w \in B\left(W_{1}\right)\right\}
$$

The axiom of choice tells us that this set exists. We need the following result. 
Lemma 3.1. Let $L: V \rightarrow V$ be a linear transformation of $V$ onto itself. Let ${ }^{m}$ be a linearly independent subset of $V$. If $S$ is a set consisting of precisely one member from each of the sets in the family

$$
\left\{L^{-1}(t): t \in T^{T}\right\}
$$

then $S$ is a linearly independent set.

Proof of Lemma 3.1. Let $v_{1}, \ldots, v_{m}$ be an arbitrary finite subset of $S$. Let $c_{1}$, $\ldots, c_{m}$ be scalars. Then $c_{1} v_{1}+\ldots+c_{m} v_{m}=0$ implies $c_{1} L\left(v_{1}\right)+\ldots+c_{m} L\left(v_{m}\right)=0$. But $\left\{L\left(v_{1}\right), \ldots, L\left(v_{m}\right)\right\}$ is an m-element subset of $T$ and is, therefore, linearly independent. Hence, $c_{1}=c_{2}=\ldots=c_{m}=0$.

Lemma 3.2. Let $L, T$, and $S$ be as defined in Lemma 3.1. If $[T]$ and $[S]$ denote the vector spaces generated by $T$ and $S$, respectively, and if $B([S])$ is a basis for $[S]$, then $L(B[S]$ is a bases for $[T]$.

Proof of Lemma 3.2. Let $\left\{u_{1}, \ldots, u_{m}\right\}$ be an m-element subset of $B([S])$. Write

$$
u_{j}=\sum_{k=1}^{p} a_{(k, j)^{v_{k}} \quad(j=1, \ldots, m)}
$$

where $\left\{v_{1}, v_{2}, \ldots, v_{p}\right\}$ is a $p$-elenent subset of $S$ and the matrix

$$
A=\left(\begin{array}{ccc}
{ }^{a}(1,1) & \cdots & a_{(1, m)} \\
\vdots & & \vdots \\
{ }^{a}(p, 1) & \cdots & a_{(p, m)}
\end{array}\right)
$$

is a cne-to-one linear transformation from m-dimensional space to $p$-dimensional space. Then $L$ is a linear implies

$$
L\left(u_{j}\right)=\sum_{k=1}^{p} a(k, j) L\left(v_{k}\right)(j=1, \ldots, m) .
$$

Now suppose that we had

$$
\sum_{j=1}^{m} c_{j} L\left(u_{j}\right)=0
$$

Then interchanging summation signs we deduce from combining 3.6 and 3.5 that

$$
\sum_{k=1}^{p}\left(\sum_{j=1}^{m} a_{(k, j)} c_{j}\right) L\left(v_{k}\right)=0
$$

But Lemma 3.1 implies that

$$
\sum_{j=1}^{m} a_{(k, j)} c_{j}=0
$$

for $k=1,2, \ldots, p$. But the fact that the matrix $A$ defined by 3.4 is one-to-one implies that 


$$
c_{1}=c_{2}=\ldots=c_{m}=n
$$

Hence, the fact that (3.6) implies (3.9) for all m-element subsets $\left\{u_{1}, \ldots, u_{m}\right\}$ of $B([S])$ tells us that $L(B[S])$ is a linearly independent set. To see that $L(B[S])$ gererates [T] we let $L(v)$ denote an arbitrary element of $T$, where $v \varepsilon S$. Then there exist $u_{1}, \ldots, u_{m}$ in $B[S]$ such that

$$
c_{1} u_{1}+\ldots+c_{m} u_{m}=v
$$

By linearity of $L$ and (3.10) we see that $L(v)$ is a linear combination of a finite number of elements in $L(B[S])$.

Let $U_{1}=W$ and let $E_{1}$ be the vector space generated by $E\left(U_{1}\right)$ and $U_{1}$, where $E\left(U_{1}\right)$ is an image of the choice function on the family of sets $\left\{L^{-1}(t): t \varepsilon B\left(W_{1}\right)\right\}$. Then we may define

$$
\mathrm{E}_{1}=U_{1} \oplus\left[E\left(U_{1}\right)\right]
$$

where [S] denotes the vector space generated by $S$ for all subsets $S$ of the vector -space V.

Lemma 3.3. Suppose $L$ is a linear transformation of $V$ into itself and $U_{1}$ is a subspace of $V$ such that $L\left(U_{1}\right)$ is a proper subspace of $U_{1}$. Suppose $E\left(U_{1}\right)$ is the set obtained by taking one member from each set in the family

$$
\left\{L^{-1}(t): t \in B\left(W_{1}\right)\right\}
$$

where $U_{1}=L\left(U_{1}\right) \oplus W_{1}$. Define

$$
E_{1}=U_{1} \oplus\left[E\left(U_{1}\right)\right]
$$

Then $E^{1}$ is a proper subspace of $E_{1}$ containing $U_{1}$ implies that $L\left(E^{1}\right)$ does not contain $U_{1}$

Proof of Lemma 3.3. Let $\pi_{1}$ be a projection of $E_{1}$ onto $\left[E\left(U_{1}\right)\right]$. Then $\pi_{1}\left(E^{1}\right)$ must be a proper subspace of $\left[E\left(U_{1}\right)\right]$ since the definition of direct sum and the fact that $E^{1}$ is a proper subspace of $E_{1}$ containing $U_{1}$ implies

$$
E^{1}=U_{1} \oplus \pi_{1}\left(E^{1}\right)
$$

We denote by $E^{1}\left(U_{1}\right)$ a basis for $\left[E\left(U_{1}\right)\right]$ which contains $B\left(\pi_{1}\left(E^{1}\right)\right)$, a basis for $\pi_{1}\left(E^{1}\right)$. By Lemma 3.2 we know that $L\left(E^{1}\left(U_{1}\right)\right)$ is a basis for $W_{1}$. Since $\pi_{1}\left(E^{1}\right)$ is a proper subspace of $\left[E\left(U_{1}\right)\right]$, it follows that $B\left(\pi_{1}\left(E^{1}\right)\right)$ must be a proper subset of $E^{1}\left(U_{1}\right)$. For if $B\left(\pi_{1}\left(E^{1}\right)\right)$ were equal to $E_{1}^{1}\left(U_{1}\right)$, then we would have

$$
\pi_{1}\left(E^{1}\right)=\left[B\left(\pi_{1}\left(E^{1}\right)\right]=\left[E^{1}\left(U_{1}\right)\right]=\left[E\left(U_{1}\right)\right]\right.
$$

which contradicts the supposition that $\pi_{1}\left(E^{1}\right)$ is a proper subspace of $\left[E\left(U_{1}\right)\right]$. Thus, (3.14) and the definition of $\pi_{1}$ imply that 


$$
L\left(E^{1}\right)=L\left(U_{1}\right) \oplus L\left(\pi_{1}\left(E^{1}\right)\right)
$$

and consequently that $L\left(E^{1}\right)$ is a proper subspace $L\left(U_{1}\right) \oplus W_{1}=U_{1}$.

Hence, $L\left(E^{1}\right)$ does not contain $U_{1}$. We use transfinite induction to coristruct for every ordinal $\alpha$ less than $\delta+1$, where $\delta$ is the ordinality of a basis for $V$, a set $E_{\alpha}$ which is minimal with respect to the property that

$$
U_{\alpha}=W \oplus\left\{U\left\{E_{\gamma}: \gamma<\alpha\right\} \subset L\left(E_{\alpha}\right)\right.
$$

and

$$
U\left\{E_{\gamma}: \gamma<\alpha\right\} \subset E_{\alpha}
$$

in the sense that if

$$
W_{\alpha} \subset E^{1} \subset \underset{\alpha}{\subset}
$$

then $L\left(E^{1}\right)$ does not contain $U_{\alpha}$. We have constructed $E_{\alpha}$ for $\alpha=1$. Thus, we suppose that $E_{\alpha}$ has been constructed for all $\beta<\alpha$. Then define $U_{\alpha}$ as in (3.16). It is clear that $U_{\alpha}$ is a vector space, since $W$ and each $E_{\gamma}$ is a vector space and $\gamma_{1}<\gamma_{2}$ implies $E_{\gamma_{1}} \subset E_{\gamma_{2}}$. Let $W_{\alpha}$ be a subspace of $V$ such that

$$
U_{\alpha}=L\left(U_{\alpha}\right) \oplus W_{\alpha} .
$$

Let $B\left(W_{\alpha}\right)$ be a basis for $W_{\alpha}$. Let $E\left(U_{\alpha}\right)$ be defined to be the set obtained by taking one element from each set in the family

$$
\left\{L^{-1}(w): W \in B\left(W_{\alpha}\right)\right\}
$$

We let $E_{\alpha}$ be the vector space generated by $U_{\alpha}$ and $E\left(U_{\alpha}\right)$. Then the Lemmas $3.1,3.2$ and the previous argument show that if $E^{1}$ satisfies $(3.18)$, then $L\left(E^{1}\right) \neq E^{1}$. Since $L(V)=V$, it is clear that there is some ordinal $\alpha_{0}$ such that $L\left(E_{\alpha_{0}}\right)=E_{\alpha_{0}}$. But the ordinals are well ordered. Thus, we may suppose

$$
\beta=\min \left\{\gamma: L\left(E_{\gamma}\right)=E_{\gamma}\right\}
$$

Indeed, it is easy to see that $\beta$ is the first infinite ordinal.

The following lemma gives important information about the space $E_{\beta}$.

Lemma 3.4. If $B$ is defined by (3.21), then $U_{B}=E_{B}$.

Proof of Lemma 3.4. Suppose $U_{\beta} \neq E_{B}$. Then $L\left(U_{\beta}\right)$ would be a proper subspace of $U_{\beta}$. It is easy to show by transfinite induction that $L\left(U_{\alpha}\right) \subset U_{\alpha}$ for every ordinal $\alpha$. Then we can write

$$
U_{B}=L\left(U_{B}\right) \oplus W_{B}
$$


Then we would let $B\left(W_{\beta}\right)$ be a basis for $W_{\beta}$ and let $E\left(U_{\beta}\right)$ be the set consisting of one member from each set in the family,

$$
\left\{L^{-1}(w): w \in B\left(W_{B}\right)\right\}
$$

Then $E_{B}$ is the vector space generated $U_{\beta}$ and $E\left(U_{\beta}\right)$ and clearly $L\left(E_{\beta}\right) \subset U_{\beta}$. This contradicts the supposition that $L\left(E_{B}\right)=E_{B}$.

Lemma 3.5. For every ordinal a less than $\delta+1$ let

$$
U_{\alpha}=\left\{E_{\gamma}: \gamma<\alpha\right\},
$$

let $W_{\alpha}$ be a subspace of $V$ such that

$$
U_{\alpha}=L\left(U_{\alpha}\right) \oplus W_{\alpha} \text {, }
$$

let $B\left(W_{\alpha}\right)$ be a basis for $W_{\alpha}$, let $E\left(U_{\alpha}\right)$ be a set consisting of precisely one element from each set in the family

$$
\left\{L^{-1}(w): W \in B\left(W_{\alpha}\right)\right\},
$$

and let

$$
\pi_{\alpha}: V \rightarrow\left[E\left(U_{\alpha}\right)\right]
$$

be a projector of $V$ onto the vector space $\left[E\left(U_{\alpha}\right)\right]$ generated by $E\left(U_{\alpha}\right)$. Then

$$
\left[E\left(U_{\alpha}\right)\right] \cap U_{\alpha}=\{0\}
$$

and for every vector $v$ in $v$

$$
v=w+\sum \pi_{\alpha}(v)(1 \leq \alpha<\delta+1),
$$

where $w$ is a member of $W$.

Proof of Lemma 3.5. To prove (3.28) note that if $v \varepsilon\left[E\left(U_{\alpha}\right)\right] \cap U_{\alpha}$ then $L(v) \varepsilon L\left(U_{\alpha}\right) \cap w_{\alpha}=\{0\}$. Thus, $v=c_{1} v_{1}+\ldots+c_{m} v_{m}$ for some elements $v_{1}, \ldots, v_{m}$ in $E\left(U_{\alpha}\right)$ and some scalars $c_{1}, \ldots, c_{m}$. But $\left\{L\left(v_{1}\right), \ldots, L\left(v_{m}\right)\right\}$ is an $m$-element subset of $B\left(W_{\alpha}\right)$, a basis for $W_{\alpha}$, and is, therefore, a linearly independent set. Hence, $L(v)=0$ implies $v=0$. In other words (3.28) is valid. Next we show that $\alpha<\beta$ implies $\pi_{\alpha}(V) \cap \pi_{\beta}(V)=\{0\}$. Now

$$
\left[E\left(U_{\alpha}\right)\right] \subset E_{\alpha} \subset U_{\beta}
$$

and by (3.28)

$$
U_{B} \cap\left[E\left(U_{B}\right)\right]=\{0\}
$$


Combining (3.30) and (3.31) we deduce that $\left[E\left(U_{\alpha}\right)\right] \cap\left[E\left(U_{\beta}\right)\right]=\{0\}$ if $\alpha$ and $\beta$ are ordinals and $\alpha<\beta$. Then (3.29) is an immediate consequence of the fact that

$$
V=W \oplus\left(\oplus \sum\left[E\left(U_{\alpha}\right)\right](1 \leq \alpha<\delta+1)\right)
$$

Proof of Theorem 3.1. Let $E^{1}$ be a proper subset of $E_{B}$ which contains $W$, where $B$ is defined by (3.21). Then $E_{\beta} \cap E^{1}=E^{1} \neq E_{B}$. Thus, we deduce that

$$
\left\{\gamma: E^{1} \cap E_{\gamma} \neq E_{\gamma}\right\} \neq \phi
$$

The well ordering property and (3.33) enable us to define

$$
\alpha=\min \left\{\gamma: E^{1} \cap E_{\gamma} \neq E_{\gamma}\right\} \text {. }
$$

From the fact that $\gamma<\alpha$ implies $E^{1} \cap E_{\gamma}=E_{\gamma}$ we deduce that

$$
U_{\alpha} \cap E^{1}=U_{\alpha}
$$

or that $U_{\alpha}$ is a subspace of $E^{1}$ if $\alpha$ is defined by (3.34). Thus, Lemma 3.5 implies that

$$
\left(\oplus \sum_{\gamma \geq \alpha} \pi_{\gamma}\left(E^{1}\right)\right)=\left(I-\tilde{\pi}_{\alpha}\right)\left(E^{1}\right)
$$

where $\tilde{\pi}_{\alpha}$ is the projection of $V$ onto $U_{\alpha}$. Furthermore,

$$
E^{1} \subset U_{\alpha} \oplus\left(I-\tilde{\pi}_{\alpha}\right)\left(E^{1}\right)
$$

But $e^{1} \varepsilon E^{1}$ implies there is a $u_{\alpha} \varepsilon U_{\alpha}$ and a $v_{\alpha} \varepsilon\left(I-\tilde{\pi}_{\alpha}\right)\left(E^{1}\right)$ such that

$$
e^{1}=u_{\alpha}+v_{\alpha}
$$

But $e^{1}-u_{\alpha} \varepsilon E^{1}$ implies $v_{\alpha} \varepsilon E^{1}$. Thus, from (3.36) we deduce that

$$
E^{1}=U_{\alpha} \oplus\left(\left(I-\pi_{\alpha}\right)\left(E^{1}\right) \cap E^{1}\right) \text {. }
$$

In order that $L\left(E^{1}\right)=E^{1}$ we must have in particular that $U_{\alpha} \subset L\left(E^{1}\right)$. But

$$
U_{\alpha}=L\left(U_{\alpha}\right) \oplus L\left(\left[E\left(U_{\alpha}\right)\right]\right)
$$

Now $\left.\left(I-\tilde{\pi}_{\alpha}\right)\left(E^{1}\right)\right) \cap E^{1}$ is a proper subspace of $\left(I-\tilde{\pi}_{\alpha}\right)(V)$. We want to show that $L\left(E^{1}\right)$ could not possibly contain $U_{\alpha}$. Suppose $L\left(E^{1}\right)$ did contain $U_{\alpha}$. Then $L\left(E^{1}\right) \cap U_{\alpha}=$ $L\left(U_{\alpha}\right) \oplus \tilde{W}_{\alpha}=U_{\alpha}$. Let $B\left(\tilde{W}_{\alpha}\right)$ be a basis for $\tilde{W}_{\alpha}$. Then $B\left(\tilde{W}_{\alpha}\right) \stackrel{\alpha}{\subset} L\left(E^{1}\right)$. Then each set in the family

$$
\left\{L^{-1}(w) \cap E^{1}: W \in B\left(\tilde{W}_{\alpha}\right)\right\}
$$


inust be nonempty. Let $E^{1}\left(U_{\alpha}\right)$ be a set consisting of one member from each set in the family (3.39). Then $\left[E^{1}\left(U_{\alpha}^{\alpha}\right)\right] \subset E^{1} \subset E_{\beta} \subset V$. Let $\gamma$ be an ordinal number larger than $\alpha$. Therefore $L\left(\left[E^{1}\left(U_{\alpha}\right)\right]\right) \subset U_{\alpha}$ and $L\left(\left[E\left(U_{\gamma}\right)\right]\right) \cap U_{\alpha}=\{0\}$. Thus, $\left[E\left(U_{\gamma}\right)\right] \cap\left[E^{1}\left(U_{\alpha}\right)\right]=$ $\{0\}$ for all ordinals $\gamma>\alpha$. Thus, $E^{1} \subset E_{B}$ implies that

$$
\left[E^{1}\left(U_{\alpha}\right)\right] \subset E^{1} \subset E_{\beta}=E_{\alpha} \oplus\left(\oplus \sum\left[E\left(U_{\gamma}\right)\right] \alpha<\gamma<\beta\right)
$$

Thus, $v \in\left[E^{1}\left(U_{\alpha}\right)\right]$ implies

$$
v=v_{\alpha}+\left(\sum v_{\gamma}, \alpha<\gamma<\beta\right) .
$$

$L\left(v-v_{\alpha}\right) \in U_{\alpha}$ and

$$
L\left(\oplus \sum v_{\gamma}, x<\gamma<\beta\right)=0
$$

or else

$$
L\left(\oplus \sum v_{\gamma}, \alpha<\gamma<\beta\right) \notin U_{\alpha}
$$

But since $L\left(v-v_{\alpha}\right) \in U_{\alpha}$, it follows that (3.43) is impossible. Hence, $L\left(v-v_{\alpha}\right) \varepsilon U_{\alpha}$. But $L$ is one-to-one on each of the subspaces $\left[E\left(U_{\gamma}\right)\right]$ and is consequently one-to-one on their direct sum. Thus, $v_{\gamma}=0$ for all ordinals $\gamma$ such that $\alpha<\gamma<\beta$, where the $v_{\gamma}$ are defined by (3.41). Hence, $v=v_{\alpha}$. This implies that $\left[E^{1}\left(U_{\alpha}\right)\right]$ is a subspace of $E_{\alpha}^{\gamma}$ and since $\left[E^{1}\left(U_{\alpha}\right)\right] \cap U_{\alpha}=\{0\}$ is consequently a subspace of $\left[E\left(U_{\alpha}\right)\right]$. But this would imply that

$$
U_{\alpha} \oplus\left[E^{1}\left(U_{\alpha}\right)\right]=E^{1} \cap E_{\alpha}
$$

is a subspace of $E_{\alpha}$ with the property that $U_{\alpha} \subset L\left(U_{\alpha} \oplus\left[E^{1}\left(U_{\alpha}\right)\right]\right)$. This implies by Lemma 3.2 that

$$
U_{\alpha} \oplus\left[E^{1}\left(U_{\alpha}\right)\right]=U_{\alpha} \oplus\left[E\left(U_{\alpha}\right)\right]
$$

Hence, $E^{1} \cap E_{\alpha}=E_{\alpha}$, which contradicts (3.34).

\section{NONUNIQUENESS OF THE MSS PROBLEM FOR THE CATEGORY}

\section{OF VECTOR SPACES AND LINEAR TRANSFORMATIONS}

In this section we prove that if $V$ is a vector space with a subspace $W$ and $L$ is an epimorphism of $V$ which maps $W$ into a proper subspace of itself, then there does not necessarily exist a unique subspace $E$ of $V$ containing $W$ such that $L(E)=E$ and $L\left(E^{1}\right) \neq$ $E^{1}$ for all proper subspaces $E^{1}$ of $E$ containing $W$. We also define the semigroup $S(V, L, W)$ of endomorphisms of $V$ which commute with $L$ and leave $W$ fixed.

Theorem 4.1. Let $F$ be a field. Then there exists a pair of vector spaces $V$ and $W$ such that both $V$ and $W$ have countable dimension, with $W$ being a subspace of $V$, such that there exists a linear transformation $L$ of $V$ onto itself which maps $W$ into but not onto itself. Furthermore, $V, W$, and $L$ described above can be constructed in such a 
way that there exists a sequence of spaces $\left\{v^{(m)}: m \in N\right\}$ such that $v=v^{(0)} \supset v^{(1)} \supset$

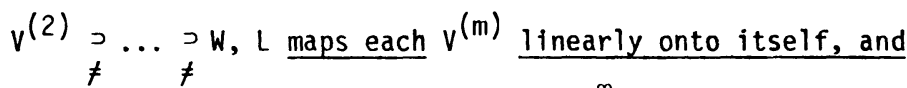

$$
\prod_{m=0}^{\infty} v^{(m)}=w
$$

In addition there exists an uncountable family of subspaces $\left\{\mathrm{E}_{\alpha}: \mathrm{a} \varepsilon \quad A\right\}$ of $V$ containing $W$ such that $L$ maps each $E_{\alpha}$ linearly onto itself and such that if $E_{\alpha}^{1}$ is a subspace of $V$ satisfying

$$
W \subset E_{\alpha \neq}^{1} \underset{\alpha}{\subset}
$$

then $L\left(E_{\alpha}^{1}\right)$ is not equal to $E_{\alpha}^{1}$. Furthermore, we may construct the minimal spaces of surjectivity $E_{\alpha}$ so that if $\alpha_{1} \neq \alpha_{2}$, then

$$
E_{\alpha_{1}} \cap E_{\alpha_{2}}=W
$$

if we ask only that $A$ be countable.

Proof. Let $v_{i}$ denote the set of all functions from $N=\{0,1,2, \ldots\}$ into $F$ which vanish for almost all members of $N$ for $i=1,2,3,4, \ldots$

Let

$$
v=v_{1} \times v_{2} \times v_{3} \times \ldots \times v_{i} \times \ldots
$$

Let

$$
W=V_{1} \times\{\underline{0}\} \times \ldots \times\{\underline{0}\} \times \ldots
$$

where $\underline{0}$ is the zero element of $v_{i}$ for $i=1,2, \ldots$ Let

$$
\gamma: N \times N \rightarrow N
$$

be a bijection. Define $L_{1}: V_{1} \rightarrow V$ by the rule,

$$
\begin{aligned}
L_{1}(v) & =\left(x_{2 N} v, \underline{0}, \underline{0}, \ldots\right) \\
& =((v(0), 0, v(2), 0, v(4), 0, \ldots), \underline{0}, \underline{0}, \ldots)
\end{aligned}
$$

Define

$$
\mathrm{L}_{2}: \mathrm{V}_{2} \rightarrow \mathrm{V} \text { by the rule, }
$$

$$
\mathrm{L}_{2}(v)=
$$

$$
\left(\left(0, \sum_{n=0}^{\infty} v(r(0, n)), 0, \sum_{n=0}^{\infty} v(r(1, n)), 0, \ldots\right), \underline{0}, \underline{0}, \ldots\right)
$$

where $\gamma$ is defined by (4.6). Define $L_{3}: V_{3} \rightarrow V$ by the rule 


$$
L_{3}(v)=(\underline{0}, v, \underline{0}, \underline{0}, \ldots) .
$$

If $m$ is an integer larger than three, define $L_{m}: V_{m} \rightarrow V$ by the rule,

$$
\begin{aligned}
L_{m}:(v)= & \left(\underline{0}, \ldots, \frac{0}{4}, v, \frac{0}{4}, \underline{0}, \ldots\right) \\
& (m-2) \text { nd mth } \\
& \text { position position }
\end{aligned}
$$

Define $L: V \rightarrow V$ by the rule,

$$
L\left(v^{(1)}, v^{(2)}, \ldots\right)=\sum_{i=1}^{\infty} L_{i} v^{(i)}
$$

Then $L$ maps $V$ linearly onto itself and $L$ maps $W$ linearly into, but not onto, itself. Define

$$
v^{(m)}=v_{1} \times v_{2}^{(m)} \times v_{3}^{(m)} \times \cdots
$$

where for each $i \varepsilon\{1,2,3, \ldots\}$,

$$
v_{i}^{(m)}=\{v \notin n+F \mid v(n)=0 \text { for } n<m\} \text {, }
$$

We first show that $L: V^{(m)} \rightarrow V^{(m)}$ is a linear mapping of $V^{(m)}$ onto itself. Let

$$
\left((v(0), v(1), v(2), \ldots), v^{(2)}, v^{(3)}, \ldots\right)
$$

denote an arbitrary member of $v^{(m)}$.

Let $P_{n}=\gamma\left(n, m_{n}\right)$, where

$$
m_{n}=\operatorname{inft} k \in N: r(n, k)>m^{3}
$$

Define

$$
w^{(1)}(2 n)=v(n) \text { for } n \in N
$$

and define

$$
w^{(1)}(2 n+1)=0
$$

for $n \in N$. Define

$$
w^{(2)}\left(P_{n}\right)=v(2 n+1) \text { for } n \in N
$$

and define

$$
w^{(2)}(k)=0 \text { if } k \notin\left\{P_{0}, P_{1}, P_{2}, \ldots\right\}
$$

Define 


$$
w^{(3)}=v^{(2)}
$$

and

$$
w^{(k+1)}=v^{(k)} \text { for } k=2,3, \ldots
$$

Then

$$
L\left(w^{(1)}, w^{(2)}, w^{(3)}, \ldots\right)=\left(v, v^{(2)}, v^{(3)}, \ldots\right)
$$

which shows that $L$ maps $V^{(m)}$ linearly onto itself. Let $\pi_{k}: V \rightarrow V_{k}$ be the natural projection.

Lemma 4.1. We may write

$$
E=\pi_{1} E \times \pi_{2} E \times \pi_{3} E \times \cdots
$$

The proof of Lemma 4.1 is immediate. Let $E_{k}=\pi_{k} E$ for $k=1,2, \ldots$. Note that $E_{2}$ and hence every $E_{k}$ must be infinite dimensional, since by hypothesis $E_{1}=V_{1}$. The proof we have just repeated shows that if we just require that $\operatorname{supp}\left(E_{2}\right)$, the support of the functions in $E_{2}$, satisfy $\operatorname{supp}\left(E_{2}\right) \cap\{\gamma(n, 0), \gamma(n, 1), \gamma(n, 2), \ldots\} \neq \emptyset$ for every $n$, and take $E_{k}=E_{2}$ for all $k=3,4,5, \ldots$ then $L$ will map $E$ linearly onto itself. Also $E$ will be minimal provided that

$$
\operatorname{suppE}_{2} \cap\{\gamma(n, 0), \gamma(n, 1), \gamma(n, 2), \ldots\}
$$

has just one element in it for every $n \in N$. There are clearly an uncountable number of ways of choosing $E_{2}$ so that the set (4.17) has just one element in it for every $n \in N$. Also, the number of ways of choosing $E_{2}$ so that condition (4.2) satisfied is at most countable since for each integer $n$ it must be true that

$$
\operatorname{supp}\left(\pi_{2} E_{\alpha_{1}}\right) \cap\{\gamma(n, 0), \gamma(n, 1), \ldots\}
$$

is not equal to

$$
\operatorname{supp}\left(\pi_{2} E_{\alpha_{2}}\right) \cap\{\gamma(n, 0), \gamma(n, 1), \ldots\} .
$$

Definition 4.1. Let $L$ be an epimorphism of a vector space $V$ which has a subspace $W$ with the property that $L(W)$ is a proper subspace of itself. Let $S(V, L, W)$ be the semi-group of all endomorphisms of $V$ which commute with $L$ and leave elements of $W$ fixed.

Theorem 4.2. If $L, V$, and $W$ satisfy the conditions of Definition 4.1 then $S(V, L, W)$ contains only one element if and only if $E$ is a proper subspace of $V$ containing $W$ implies $L(E) \neq E$.

Proof. Suppose $E$ were a subspace of $V$ containing $W$. Let us write $V=\operatorname{Ker}(L) \oplus F$ and write $E=\operatorname{Ker}(L) \cap E \oplus F$. Let $B(F)$ be a basis for $F$, and let $B(F)$ be a basis for $F$ containing $B(\tilde{F})$. Let $B(\operatorname{Ker}(L))$ be a basis for $\operatorname{Ker}(L)$ containing $B(\operatorname{Ker}(L) \cap E)$, a basis for $\operatorname{Ker}(L) \cap E$. Then each $v$ in $V$ may be written as 


$$
\begin{aligned}
v= & a_{1} \tilde{w}_{1}+\ldots+a_{p} \tilde{w}_{p}+b_{1} w_{1}+\ldots+b_{q} w_{q} \\
& +c_{1} \tilde{u}_{1}+\ldots+c_{r} \tilde{u}_{r}+d_{1} u_{1}+\ldots+d_{s} u_{s}
\end{aligned}
$$

where

$$
\begin{gathered}
\left\{\tilde{w}_{1}, \ldots, \tilde{w}_{p}\right\} \subset B(\operatorname{Ker}(L) \cap E), \\
\left\{w_{1}, \ldots, w_{q}\right\} \subset B(\operatorname{Ker}(L))-B(\operatorname{Ker}(L) \cap E), \\
\left\{\tilde{u}_{1}, \ldots, \tilde{u}_{r}\right\} \subset B(\tilde{F}),
\end{gathered}
$$

and

$$
\left\{u_{1}, \ldots, u_{s}\right\} \subset B(F)-B(\tilde{F})
$$

Then define

$$
\pi(v)=a_{1} \tilde{w}_{1}+\ldots+a_{p} \tilde{w}_{p}+c_{1} \tilde{u}_{1}+\ldots+c_{r} \tilde{u}_{r}
$$

We deduce from the definition (4.23) of $\pi$ and the fact that $L(E)=E$, that

$$
L(\pi(v))=c_{1} L\left(\tilde{u}_{1}\right)+\ldots+c_{r} L\left(\tilde{u}_{r}\right)=\pi(L(v))
$$

Thus, the projector $\pi$ we have constructed belongs to $S(V, L, W)$. Conversely, if $\psi$ belongs to $S(V, L, W)$ then $E=\psi(V)$ satisfies $L(E)=L(\psi(V))=\psi(L(V))=E$. It is clear that the identity transformation I belongs to $S(V, L, W)$. From our construction it is clear that if $E$ is a proper subspace of $V$ such that $L(E)=E$, then the projector $\pi$ we have constructed is distinct from the identity. This completes the proof of the Theorem.

5. SURJECTIVITY OF DIFFERENTIAL OPERATORS ON LOCALLY CONVEX

SPACES CONTAINING THE MEROMORPHIC FUNCTIONS

Let $R\left(C^{n}\right)$ denote the meromorphic functions of $n$ complex variables. We construct a special locally-convex space $E^{(n)}$ containing $R\left(C^{n}\right)$ such that every nontrivial linear partial differential operator with $n$ independent variables and constant coefficients maps $E^{(n)}$ continuously onto itself.

For each $j \in\{1, \ldots, n\}$ and for each $u(x) \in C\left[\left[x_{1}, \ldots, x_{n}\right]\right]$ define

$$
T_{j} u(x)=u\left(x_{1}, \ldots, x_{j-1}, 1-x_{j}, x_{j+1}, \ldots, x_{n}\right) .
$$

Let $F^{(n)}=\sum_{\gamma} E_{\gamma}^{(n)}$ (exterior direct sum), where $\gamma$ runs through the set $I$, where

$$
I=\{0,1\} \times \ldots \times\{0,1\} \quad \text { (n factors) }
$$

where 


$$
\begin{aligned}
& E_{0}^{\left(n_{1}\right)}=c\left[\left[x_{1}, \ldots, x_{n}\right]\right], \\
& E_{\gamma}^{(n)}=T_{1}^{\gamma} \ldots T_{n}^{\gamma} c\left[\left[x_{1}, \ldots, x_{n}\right]\right],
\end{aligned}
$$

and we agree that $T_{j}^{0}=I$, the identity map on $c\left[\left[x_{1}, \ldots, x_{n}\right]\right]$. These spaces of formal power series are equipped with the usual locally-convex topology of simple convergence of coefficients.

Proposition 1. The dual space of $\mathrm{E}_{\gamma}^{(n)}$ is isomorphic to the space $C\left[T_{1}^{\gamma} 1_{X_{1}}, \ldots, T_{n}^{\gamma} n_{X_{n}}\right]$ of polynomials. Furthermore, $E_{\gamma}^{(n)}$ is a reflexive Frechet space for all $\gamma$ in $I$, where $I$ is given by (5.2).

Proof. It is well-known (e.g. Treves [2], page 266) that $E_{0}^{(n)}$ and $C\left[x_{1}, \ldots, x_{n}\right]$ are duals of one another. But if $E^{\prime} \subset E$, where $E$ is a topological vector space, and $J: E \rightarrow F$ is an isomorphism then $F^{\prime}=J\left(E^{\prime}\right)$. Furthermore, if $E$ is a Frechet space, $F$ is a locally convex space and $\mathrm{J}: E \rightarrow F$ is a topological isomporphism, then $F$ is also a Frechet space.

- Definition 1. The duality bracket between a polynomial $P$ in $C\left[x_{1}, \ldots, x_{n}\right]$ and a formal power series $u$ in $c\left[\left[x_{1}, \ldots, x_{n}\right]\right]$ is given by

$$
\left\langle P, u>=\sum_{\alpha \in N} n\left(\frac{1}{\alpha !}\right)\left[(\partial / \partial X)^{\alpha} P(X)\right]_{X=0}\left[(\partial / \partial X)^{\alpha} u(X)\right]_{X=0}\right. \text {. }
$$

The duality bracket between a polynomial $Q_{\gamma}$ in the dual of $E_{\gamma}^{(n)}$ and a formal power series $v_{\gamma}$ in $E_{\gamma}^{(n)}$ is given by

$$
\left\langle Q_{\gamma}, v_{\gamma}>=\sum_{\alpha \in N} n\left(\frac{1}{\alpha !}\right)\left[(\partial / \partial X)^{\alpha} Q_{\gamma}\right]_{X=\gamma}\left[(\partial / \partial X)^{\alpha} v_{\gamma}\right]_{X=\gamma} .\right.
$$

It is easy to see that if

$$
P(\partial / \partial X)=\sum_{\alpha \varepsilon N} n(1 / \alpha !)\left[(\partial / \partial X)^{\alpha} P(X)\right]_{X=\gamma}(\partial / \partial X)^{\alpha}
$$

and $u \in E_{0}^{(n)}$, then

$$
\langle P, u\rangle=[P(\partial / \partial X) u]_{X_{i}=\gamma_{i}} \quad i \varepsilon\{1, \ldots, n\}
$$

It is similarly easy to check that if

$$
Q_{\gamma}(\partial / \partial X)=\sum_{\alpha \varepsilon N} n(1 / \alpha !)\left[(\partial / \partial X)^{\alpha} T^{\gamma} Q_{\gamma}(X)\right]_{X=\gamma}(\partial / \partial X)^{\alpha}
$$

and $v_{\gamma} \in E_{\gamma}^{(n)}$, then

$$
\left\langle Q_{\gamma}, v_{\gamma}>=\left[Q_{\gamma}(\partial / \partial X) v_{\gamma}\right]_{X_{j}}=\gamma_{i} \quad i \varepsilon\{1, \ldots, n\}\right.
$$

By E. Borel's Theorem (e.g. Treves [3], Theorem 18.1) if $u$ belongs to $E_{0}^{(n)}$, there is a $\phi$ in $C_{0}^{\infty}\left(R^{n}\right)$ such that the coefficients of the Taylor series expansion for $\phi$ about 
$x^{(0)}=(0, \ldots, 0)$ are identical to the coefficients of $u$. Thus, we may rewrite (7) as

$$
\langle P, u\rangle=\langle P(-\partial / \partial X) \delta, \phi\rangle,
$$

where $\delta$ is the Dirac delta function. Again by E. Borel's Theorem (e.g. Treves [3], Theorem 18.1) there is for every $v_{\gamma}$ in $E_{\gamma}^{(n)}$ a $\phi_{\gamma}$ in $C_{0}^{\infty}\left(P^{n}\right)$ such that the coefficients of the Taylor series expansion of $\phi^{(j)}$ about $\gamma$ are equal to the corresponding coefficients of $v_{j}$, where $X^{(\gamma)}=\gamma$. Thus, we may rewrite (5.9) as

$$
\left\langle Q_{\gamma}, v_{\gamma}\right\rangle=\left\langle Q_{\gamma}(-\partial / \partial X) \delta(X-X(\gamma)), \phi_{\gamma}>\right.
$$

Let $L=L(\partial / \partial X)$ denote a linear partial differential operator with constant coefficients. We can use $m(5.10)$ and $(5.11)$ to determine the action to $t_{L}$ on $E_{0}^{(n)}$, and $E_{\gamma}(j)$, for all $\gamma$ in $I$, where $I$ is given by $(5.2)$. It is well known that

$$
\langle P, L(\partial / \partial X) U\rangle=\langle P(-\partial / \partial X) \delta, L(\partial / \partial X) \phi\rangle
$$

implies that the transpose of $L$ is one-to-one, since it follows that

$$
t_{L P(X)}=L(x) P(x) \text {. }
$$

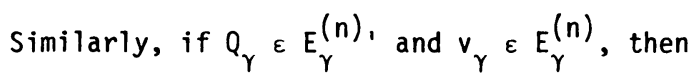

$$
\left\langle Q_{\gamma}, L(\partial / \partial X) U>=\left\langle L(-\partial / \partial X) P(-\partial / \partial X) \delta, \phi_{\gamma}\right\rangle\right. \text {. }
$$

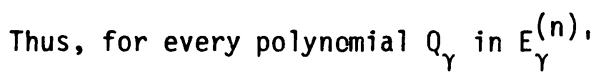

$$
t_{L Q_{\gamma}}=L\left(T_{1}^{\gamma} x_{1}, \ldots, T_{n}^{\gamma_{n}} x_{n}\right) Q_{\gamma}
$$

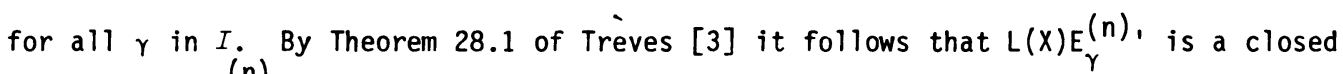
subspace of $E_{\gamma}^{(n)}$, for every $\gamma$ in $I$. This, in view of a classical theorem due essentially to $S$. Banach, which states that a continuous linear map $L$ of one Frechet space $E$ into another Frechet space $F$ is an epimorphism if and only if its transpose is one-to-one and weakly closed, implies that $L: E_{\gamma}^{(n)} \rightarrow E_{\gamma}^{(n)}$ is an isomorphism for all $\gamma$ in $I$.

Let $v_{\gamma}^{(n)}$ be the subspace of $E_{\gamma}^{(n)}$ consisting of all members of $E_{\gamma}^{(n)}$ which may be identified with a member of $\sum_{\gamma^{\prime} \neq \gamma} E_{\gamma^{\prime}}^{(n)}$ (exterior direct sum). Then $L$ is still an epimorphism of $E_{\gamma}^{(n)} / V_{\gamma}^{(n)}$ since $L\left(V_{\gamma}^{(n)}\right) \subset v_{\gamma}^{(n)}$. Note that $v_{\gamma}^{(n)}$ is closed. The space $R\left(C^{n}\right)$ can be identified with a subspace of

$$
E^{(n)}=E_{n}^{(n)} / V_{n}^{(n)} \oplus \ldots \oplus E_{1}^{(n)} / V_{1}^{(n)} \oplus E_{0}^{(n)}
$$

Thus, $R\left(C^{n}\right)$ can, when regarded as a vector space, be given a locally convex topology in a natural way, namely the one induced by $E^{(n)}$.

Let $f\left(z_{1}, \ldots, z_{n}\right) / g\left(z_{1}, \ldots, z_{n}\right)$ be a member of $R\left(c^{n}\right)$. Let $\pi_{j}: c^{n} \rightarrow c^{n-1}$ be defined by the rule, 


$$
\pi_{j}(z)=\left(z_{1}, \ldots, z_{j-1}, z_{j+1}, \ldots, z_{n}\right)
$$

Let us write

$$
g\left(z_{1}, \ldots, z_{n}\right)=g_{0}\left(\pi_{j}(z)\right)+z_{j} g_{1}\left(\pi_{j}(z)\right)+\ldots+z_{j}^{r} g_{r}\left(\pi_{j}(z)\right)+\ldots .
$$

Let $r$ be the smallest positive integer such that $g_{r}\left(\pi_{j}(z)\right) \neq 0$. Then

$$
\begin{aligned}
\frac{1}{g(z)} & =\frac{1}{z_{j}^{r}} \sum_{k=0}^{\infty} h_{k}(\pi j(z)) z_{j}^{k} \\
& =\frac{h_{0}\left(\pi_{j}(z)\right)}{z_{j}^{r}}+\ldots+\frac{h_{r-1}\left(\pi_{j}(z)\right)}{z_{j}}+\sum_{k=r}^{\infty} h_{k}(\pi j(z)) z_{j}^{k-r},
\end{aligned}
$$

where the functions $h_{k}(\pi j(z))$, are members of $R\left(c^{n-1}\right)$. Proceeding in this manner we deduce that each representative of a member of $R\left(C^{n}\right)$ is contained in $\sum_{\gamma \varepsilon} T^{\gamma} C\left[\left[x_{1}, \ldots, x_{n}\right]\right]$ (exterior direct sum) or eliminating redundancy that

$$
R\left(C^{n}\right) \subset C[[x]] \oplus \sum_{\substack{\gamma \varepsilon \\ \gamma \neq 0}} T^{\gamma} c[[x]] / K_{\gamma}^{(n)} .
$$

REFERENCES

[1] Northcott, D. G., Homological Algebra, Cambridge: University Press, (1960).

[2] Treves, J. F., Linear Partial Differential Equations with Constant Coefficients, New York: Gordon and Breach, (1966).

[3] Treves, J. F., Topological Vector Spaces Distributions and Kernels, New York: Academic Press, (1967).

[4] Treves, J. F., Applications of Distributions to PDE Theory, American Math. Monthly 77, (1970), 241-248. 


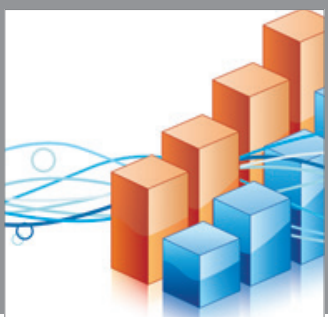

Advances in

Operations Research

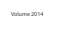

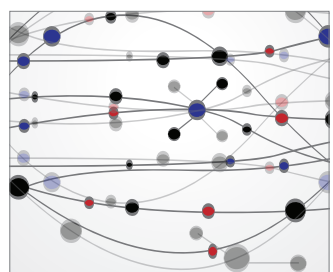

\section{The Scientific} World Journal
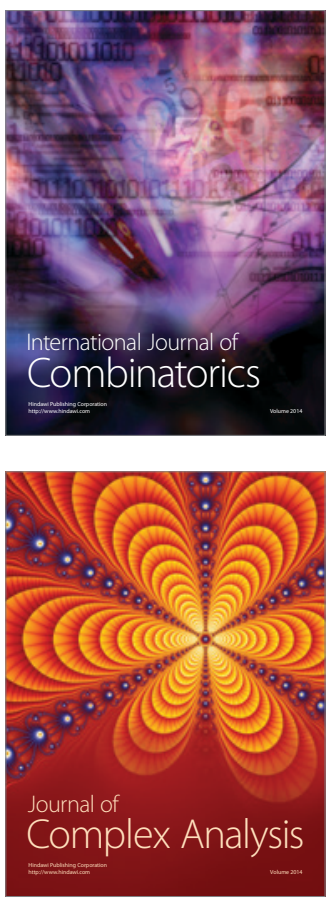

International Journal of

Mathematics and

Mathematical

Sciences
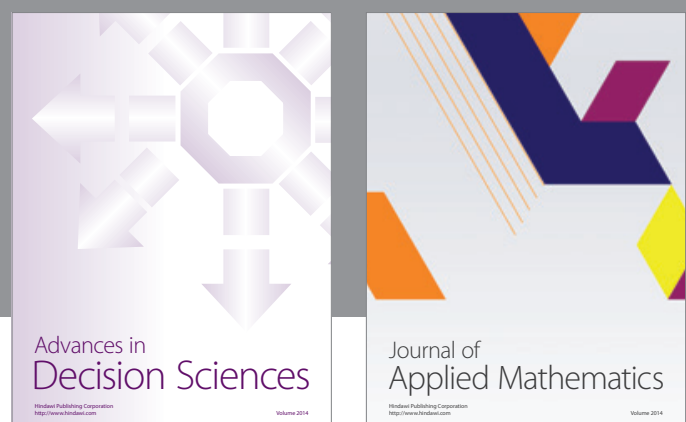

Journal of

Applied Mathematics
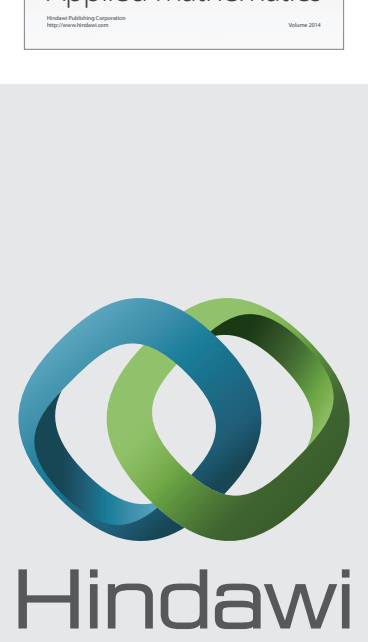

Submit your manuscripts at http://www.hindawi.com
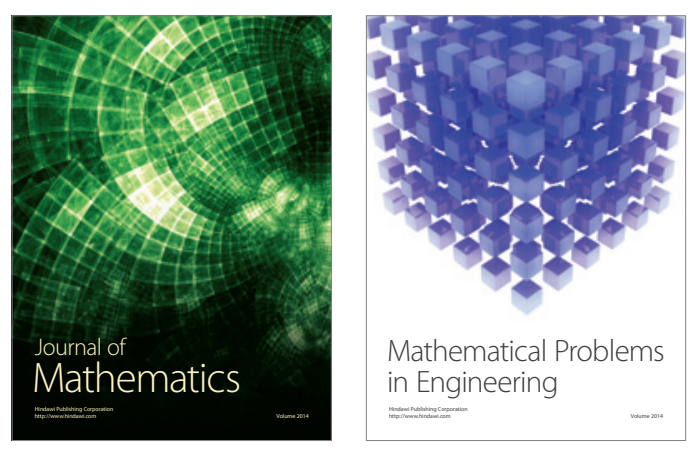

Mathematical Problems in Engineering
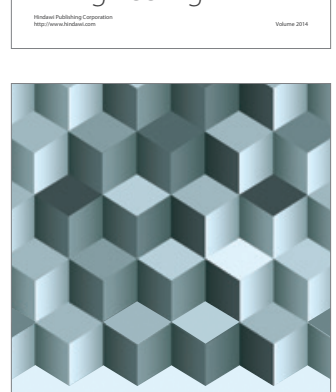

Journal of

Function Spaces
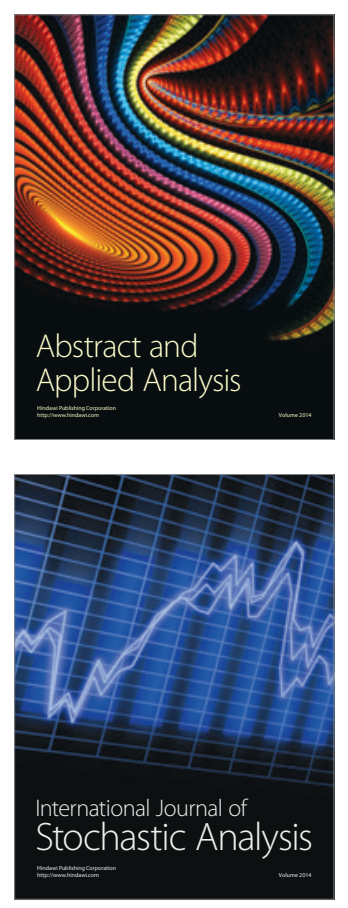

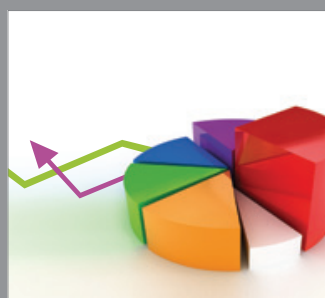

ournal of

Probability and Statistics

Promensencen
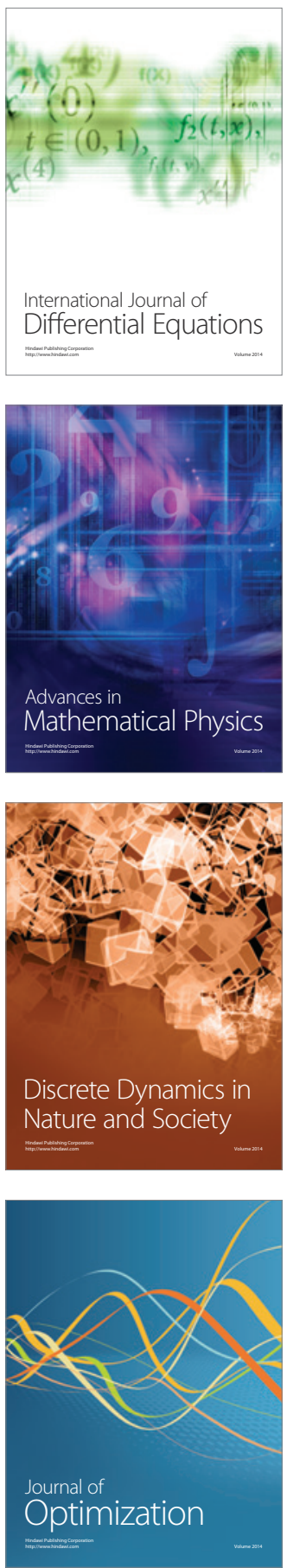